\title{
Strain Mapping on Semiconductor Device by Dark Field Electron Holography
}

\author{
Y.Y. Wang, J. Li, J. Bruley, and A. Domenicucci, D. Cooper* and R. Jean-Luc* \\ IBM Micro-Electronics Division, 2070 Route 52, Hopewell Junction, NY 12570 \\ *CEA LETI-Minatec, 17, Rue des Martyrs, F38054 Grenoble, France
}

Recent development of CMOS Si semiconductor device shows that strain along the channel region greatly enhances the device performance, which has assisted further scaling of semiconductor devices. For PFET device, the compressive stress is beneficial to the device performance, while for NFET device, the tensile stress is. Because of that, the strain measurement at nano-meter scale becomes critical for device engineering for next generation integrated circuit (IC) development and manufacturing.[1]

It has been shown that converging beam and nano-beam diffraction can be used to measure strain of Si.[1] Recently, Mytch et al. reported that dark field holography provided a promising path to a nanometer scale strain map.[2] Initial strain map by Mytch et al. was done on FEI instrument with a Lorentz lens (single lens holography), which has fringe spacing about $2.0 \mathrm{~nm}$ (4 nm spatial resolution). The principle of dark field holography is to use a biprism to overlap a strained region of the device with an unstrained region in $\mathrm{Si}$, as shown in figure 1. By selecting a specific diffracted beam with an objective aperture to obtain a dark field hologram, the difference of lattice constant between strained and unstrained Si can be measured and the change of the lattice constant can be mapped out through a data processing.[2]

In this paper, we show that using a dual lens system on JEOL 2010F2, we can achieve a fringe spacing relative to the sample down to $1.0 \mathrm{~nm}$ ( $2 \mathrm{~nm}$ spatial resolution), thus, provide a more detailed map of strain distribution. In a previous paper,[3] we developed a dual lens system for electron holography with an adjustable fringe spacing (related to spatial resolution) and fringe width (field of view) by adjusting relative strength of an objective lens and an objective mini-lens to achieve different magnification of the image at the image plane relative to the biprism. In this case, we set the objective lens at $5.5 \mathrm{~V}$, with fringe spacing and fringe width as of $1.0 \mathrm{~nm}$ and $200 \mathrm{~nm}$, respectively. The device has $17 \%$ Ge concentration in e-SiGe.

Dark field holography was obtained by selecting (220) diffracted beam. Hologram was taken on a $2 \mathrm{k} x 2 \mathrm{k}$ GIF system. Data processing was done according to the similar procedure as in the published paper by Hytch et al.[2] Figure 2 shows the change of lattice constant map along (220) direction. The red color shows compressive lattice constant and blue color shows expansion. The strain map provides a detailed strain distribution with high spatial resolution. Figure 3 is a diagram of strain distribution due to e-SiGe on both side of device (Ge atom is larger than $\mathrm{Si}$ atom; therefore, e-SiGe provides a compressive strain to the channel in the middle).

From the map, the compressive strain from both side of e-SiGe is clearly shown, which matches with simulation. The strain is weaker in the middle of the channel comparing with strain near the boundary of $\mathrm{Si}$ and e-SiGe. From the map, one can realize that by reducing the channel width to bring two boundaries closer, one can enhance the strain in the middle of the channel region to increase carrier mobility. This has been confirmed by electric measurement of a device with a closer e-SiGe design. 
Figure 4 shows a vertical profile of strain with an average parallel to Si surface as of a blue box in figure 2 . It shows that at the surface of $\mathrm{Si}$, the strain is the highest, with $-0.5 \%$ lattice constant change along (220) direction.

In conclusion, we reported that dark field holography with dual lens operation provides a high spatial resolution strain map, which is critical for strain engineering to enhance device performance.

\section{Reference:}

1. J. Li, A. Domenicucci, D. Chidambraaro, B. Greene, N. Rovedo, J. Holt, D. Dunn, K, Rim and H. Ng. Materials Research Society Symposium Proceedings, v 913, Transistor ScalingMethods, Materials and Modeling, 2006, p 157-162

2. M. Hytch, F. Houdellier, F. Hue, E. Snoeck, Nature, Vol. 453, P. 1086, (2008)

3. Y.Y. Wang, M. Kawasaki, J. Bruley, M. Gribelyuk, A. Domenicucci, J. Gaudiello, Ultramicroscopy, 101, pp.63-72 (2004); US patent \#: US 7,015,469 B2 (2006).

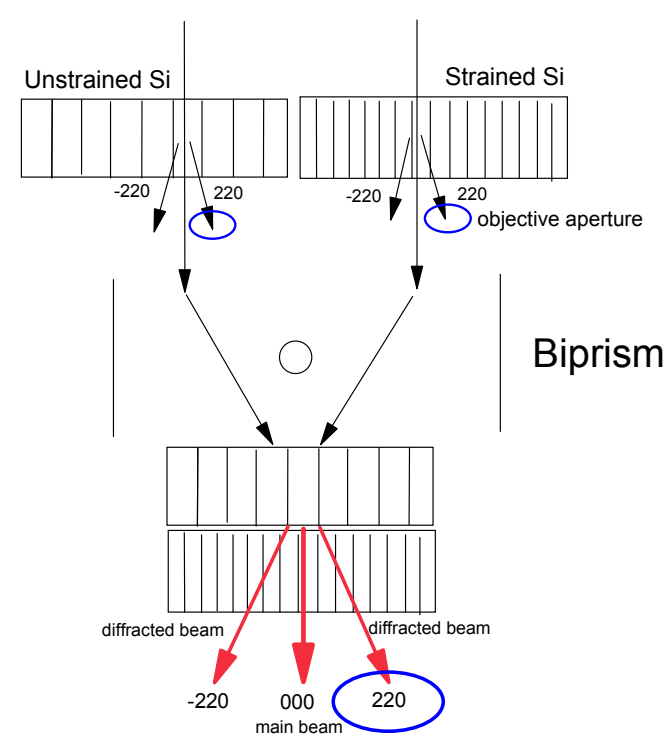

Fig. 1: Diagram of dark field holography

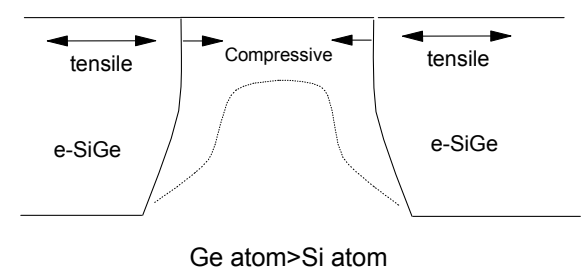

Fig.3: Diagram of e-SiGe device

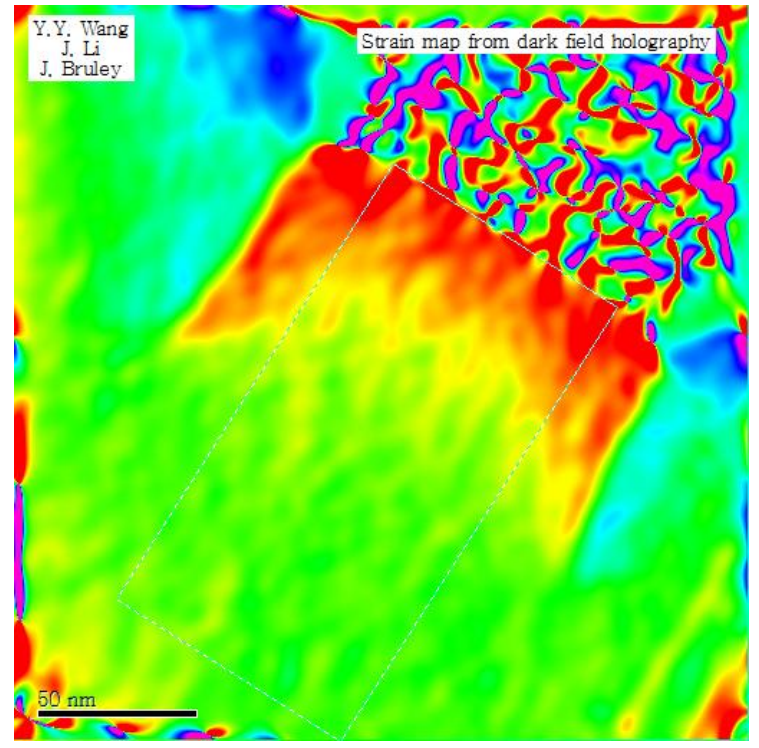

Fig.2: Lattice constant map of e-SiGe device

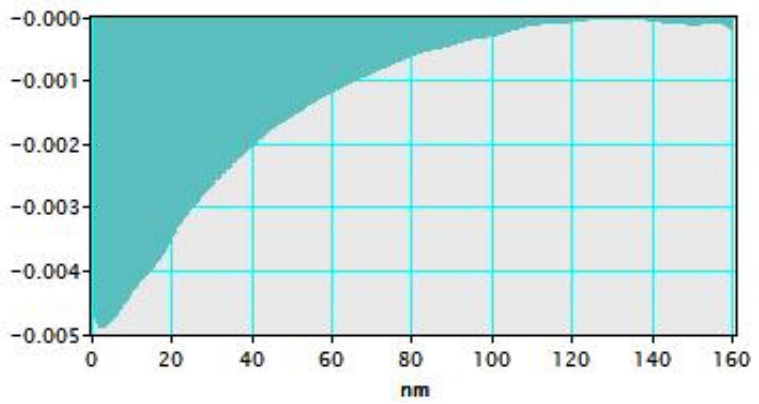

Fig.4: Vertical profile of lattice constant in the channel region from the map. Zero is the top surface of Si. 